\title{
Dessine-toi un modèle! Récit de pratique
}

\author{
Johanne Rocheleau ${ }^{\mathrm{a}}$
}

RÉSUMÉ. Cet article est un récit de pratique qui relate la coconstruction d'un modèle d'une démarche éthique, élaboré par un groupe de praticiens et de professeurs, raconté du point de vue de la personne qui a amorcé la modélisation de cette démarche. Sans connaissance en éthique, mais spécialiste en design pédagogique et en ergonomie cognitive des interfaces d'apprentissage, et dans les faits, gestionnaire d'un service public, l'auteure a utilisé un outil de sa pratique quotidienne : la technique de modélisation par objets typés. Elle a ainsi représenté sa compréhension des discussions de la communauté de pratique et partagé sa vision avec les autres membres pour mieux comprendre les dimensions et les composantes d’une démarche éthique. Il en est ressorti un modèle commun qui peut soutenir le gestionnaire dans sa pratique et l'aider à considérer tous les éléments et les angles avant de prendre des décisions pour l'action.

ABSTRACT. This article is a story of practice that presents the co-construction of an ethical approach model, developed by a group of practitioners and teachers, from the point of view of the person who started modeling this approach. Without special knowledge of ethics, but being a specialist of educational design and cognitive ergonomics of learning interfaces, and in fact, manager of a public service, the author used a tool of her daily practice: the modeling technique by typed objects. She represented her understanding of the community of practice's discussions, and shared her vision with other members to better understand the dimensions and components of an ethical approach. This has resulted in a common model that can support the manager in his practice and help him to consider all elements and angles for decision-making.

Dans cet article, je vais raconter, d'une manière très personnelle, la passionnante histoire de l'élaboration d'un modèle d'une démarche éthique qui s'est déroulée, pour ma part, de 2013 à 2018. Après avoir décrit le contexte et les acteurs de cette démarche, j’expliquerai sommairement les éléments génériques qui permettent de représenter cette démarche par la technique de modélisation par objets typés. J'aborderai ensuite la coconstruction de ce modèle d'une démarche éthique de manière chronologique et vous présenterai la version finale du modèle qui, dans ma pratique professionnelle, me sert de balises et de grille d'analyse pour gérer des services publics au quotidien.

\section{Présentation et contexte}

Designer pédagogique de cours en ligne, spécialiste en ergonomie cognitive, j'ai été professeure et chercheure universitaire pendant plusieurs années. Je gère maintenant des projets d'envergure en développement de formation tout au long de la vie (qu'on appelait auparavant «formation continue »). Dans ce cadre, je développe des programmes et des interfaces d'apprentissage synchrones et asynchrones dans lesquels je déploie, avec mon équipe, des stratégies d'enseignement et d'apprentissage permettant de mieux percevoir, traiter, encoder, mémoriser et recouvrer des savoirs, des savoir-faire, des savoir-être et des savoirs agir. Bref, j'évolue dans le développement des compétences réelles d'étudiants de l'enseignement supérieur dans un univers à la fois présentiel (en classe) et virtuel (en ligne).

Ces stratégies d'enseignement et d'apprentissage prennent plusieurs formes. L'une d'entre elles est la modélisation par objets typés, qui consiste à élaborer des cartes conceptuelles afin de représenter - par des formes et des liens - les objets typés, l'articulation de ces savoirs. J'utilise cette stratégie depuis 1994, alors que je travaillais au projet de recherche-action « L'école

${ }^{a} \mathrm{Ph}$. D., Directrice adjointe responsable de la formation continue et des services à la communauté, Collège Dawson 
informatisée Clés en mains» au Centre de recherche LICEF de la Télé-Université, qui visait, entre autres, à élaborer le modèle de la démarche d'informatisation que nous avons réalisée dans deux écoles secondaires.

Beaucoup plus tard, de 2013 à 2015, j'ai réalisé un mandat pour le Centre des Premières Nations Nikanite de l'Université du Québec à Chicoutimi. Il s'agissait de développer un Programme court de premier cycle en relation d'aide (0139) pour des étudiants autochtones répartis géographiquement sur le territoire québécois. Dans ce programme, le cours 4ETH124 Enjeux éthiques de la pratique professionnelle fut alloué à monsieur Marc Jean. On a donc développé ce premier cours en ligne de concert dans ce contexte. Des boutades, des rigolades et une franche camaraderie dans un processus de travail exigeant nous ont poussés vers une collaboration à plus long terme.

En effet, à l'hiver 2015, une chose en entraînant une autre, monsieur Jean m'a demandé de développer un programme court de deuxième cycle en éthique à être offert en ligne à des étudiants québécois et français, en reprenant le format utilisé pour le développement du programme 0139, mais en y ajoutant un volet d'enseignement collaboratif Québec-France. C'est dans ce contexte que j'ai fait la connaissance de monsieur Didier Benoit et nous avons ensemble développé trois activités universitaires passionnantes de 45 heures chacune, avec des études de cas hilarantes, mais drôlement structurantes et pleines de rebondissements!

\section{Un premier pied dans le bain de l'éthique}

L'atmosphère conviviale s'est maintenue, si bien que je me suis tournée naturellement vers Marc et Didier (eh oui! Les « monsieurs » sont disparus!) lorsque j’ai rencontré des problèmes d'éthique dans le cadre de mon implication bénévole au sein d'organismes communautaires. Je leur ai écrit un courriel en mai 2015 décrivant ce que je ressentais, cherchant du coup leurs points de vue sous l'angle de l'éthique. C'est à ce moment que je prononce mes premiers mots en matière d'éthique : malaise, devoir, légitimité, valeur, conflit, contradiction, non-dits, etc. Le malaise que je ressens est si grand! Pour moi, c'est à ce moment que l'éthique est devenue réelle et je la ressentais comme telle, comme lorsqu'on plonge un premier pied dans une baignoire d'eau chaude. Depuis l'éthique n'est plus théorique; elle est physique, tangible et pratique et je la ressens.
Mon éthique m'a fait mal, et Marc et Didier m'ont aidée à la guérir. Il y eu suffisamment d'échanges entre nous trois concernant les problèmes rencontrés que lorsqu'est venue à Didier et Marc l'idée de fonder une communauté de pratique éthique France-Québec, ils m’ont incluse dans le groupe, avec des étudiants impliqués dans la pratique éthique depuis beaucoup plus longtemps que moi. Mon intérêt était tout là!

\section{La douche froide de l'éthique}

Dans le groupe québécois, nous allions nous réunir cinq fois en conférence Web au cours de l'année scolaire 2015-2016. À chaque réunion, nous partagions nos expériences, tentions d'y donner un sens, de nommer ce que nous ressentions et nous explorions des pistes de résolution de problèmes en nous donnant des balises du domaine de l'éthique. Dès la première rencontre, j’ai dû faire quelques constats concernant ma situation et les acquis des autres membres de la communauté de pratique :

- Ils avaient tous lu quelques textes du philosophe Paul Ricœur (1990). Pas moi.

- Ils avaient tous déjà analysé de nombreux problèmes éthiques. Pas moi.

- Ils avaient tous étudié avec Marc ou Didier. Pas moi.

J'ai presque toujours été présente, mais je n'ai pu participer à la deuxième rencontre. Au début, je ne comprenais pas grand-chose, mais j'écoutais attentivement, je prenais des notes et surtout j'ai relu plusieurs fois les comptes rendus des premières réunions rédigés par Marc. Lors de la quatrième rencontre, puisque je ne comprenais toujours pas, j’allais baisser les bras, lorsque je me suis dit: «Dessine-toi donc un modèle! » C'est ce que j'aurais conseillé à un étudiant. Pourquoi ne pas le faire pour moi-même? C'est ainsi, en janvier 2016, que j'ai commencé à dessiner ma compréhension d'une démarche éthique pour mon bénéfice personnel, en utilisant la modélisation par objets typés.

\section{Le glossaire de la modélisation par objets typés}

L'outil logiciel que j'utilise pour créer les modèles de connaissances est Mot Plus (modélisation par objets typés et plus), un logiciel développé dans les années 
90 au Centre de recherche LICEF de la Télé-Université, qui est encore offert gratuitement sur le $\mathrm{Web}^{1}$. Pour que je vous partage mes dessins ou modèles, il est important d'expliquer d'entrée de jeu ce que signifient les formes des connaissances et les liens qui les unissent dans la technique de modélisation par objets typés. C'est ce qu'on appelle le « glossaire de la modélisation par objets typés ». Voici un tableau qui liste les 4 formes de connaissances principales :

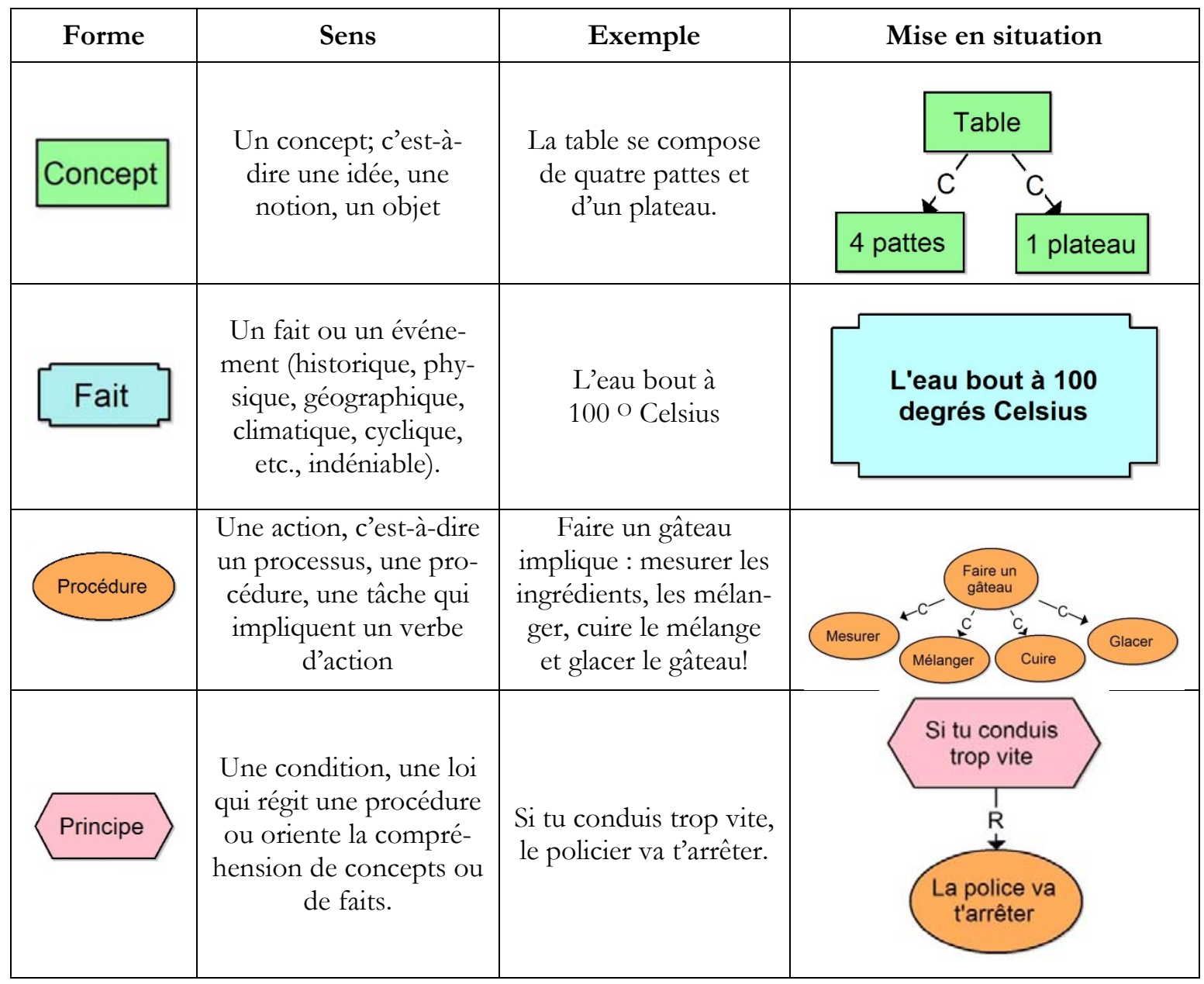

Tableau 1 - Les formes de connaissances

On a donc, dans le tableau 1, quatre connaissances qui sont « concept, fait, procédure et principe » :

- Un concept est une convention qui permet de nous entendre et un savoir factuel. On a convenu collectivement d'appeler cet objet « table », et malgré divers modèles de tables, nous sommes capables de nous comprendre;

- Un fait est évidemment un savoir factuel, mais souvent aussi une réalité partagée. Par exemple, c'est un fait que la bataille des Plaines d'Abraham a eu lieu en 1759;

- Une procédure est une action dans laquelle nous démontrons notre savoir-faire et notre savoir-être, comme lorsque nous faisons un gâteau. Une procédure est habituellement un élément d'un processus et elle se décline en tâches, qui à leur tour se divisent en activités;

- Un principe est une compréhension supplémentaire qui régit le savoir agir. Il précise les conditions ou le contexte de l'action. Par exemple : Si la température intérieure de la salle est inférieure à 20 degrés Celsius, alors mettez en marche le dispositif de chauffage.

Voici maintenant un exemple (figure 1); le processus Corriger les examens, lequel présente les différentes formes et liens qui les unissent: 


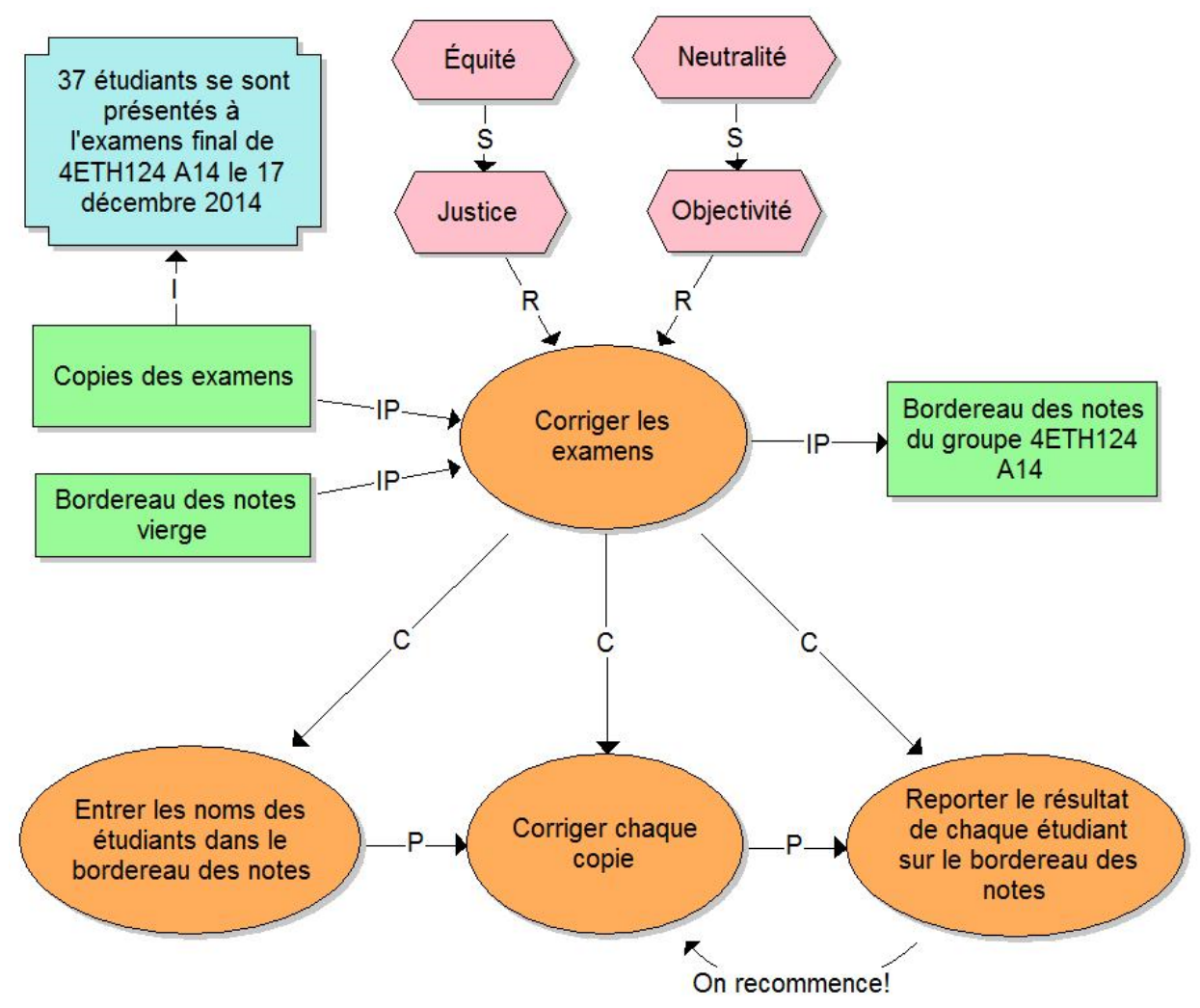

Figure 1 - Un exemple d'articulation des connaissances et les liens qui les unissent

Pour le processus Corriger les examens, il nous faut les Copies des examens et le Bordereau vierge des notes qui sont des concepts intrants (IP). Le lien IP signifie Intrant ou Produit. En fait, un Intrant est ce qui est requis ou contribue à réaliser une tâche, alors qu'un Produit est le résultat d'une tâche. C'est le sens de la flèche qui détermine l'Intrant (qui va vers la procédure) et le Produit (qui sort de la procédure). Dans cet exemple, il y a 37 copies puisqu'on voit le Fait que 37 étudiants se sont présentés à l'examen final de 4ETH124 A 14 le 17 décembre 2014, qui est relié par un lien I signifiant Instantiation (c'est-à-dire dans l'Instance, 37 étudiants se sont présentés). Le processus Corriger les examens se Compose $(\mathbf{C})$ donc des procédures :

- Entrer les noms des étudiants dans le bordereau de notes, qui précède (lien $\mathbf{P}$ );

- Corriger chaque copie, qui précède (lien $\mathbf{P}$ );

- Reporter le résultat de chaque étudiant sur le bordereau des notes.

Le Produit (IP) de Corriger les examens est le Bordereau des notes du groupe 4ETH124 de l'automne 2014.
Dans l'exemple (figure 1), on constate que les principes de Justice et d'Objectivité Régulent (lien R) (ou Régissent) le processus Corriger les examens. Le principe d'Équité est une Sorte (S) de principe de Justice, tout comme la Neutralité est une Sorte (S) d'Objectivité.

En principe, on devrait pouvoir systématiser toutes les connaissances avec la technique de modélisation par objets typés, mais il m'arrive très souvent d'en transcender la grammaire pour arriver à mes fins. Cette technique est importante pour mon travail et ma gestion de programmes et de projets en design pédagogique, de même que pour articuler les divers types de connaissances et contribuer au développement des connaissances des étudiants. Il n'est cependant pas nécessaire que vous la maîtrisiez pour comprendre le modèle d'une démarche éthique. On comprend logiquement la démarche sans avoir à apprendre le glossaire de la modélisation par objets typés par cœur. 


\section{Un premier modèle personnel}

Face aux autres membres de la communauté de pratique éthique, je me trouvais démunie et encore une fois, je ressentais un malaise. J'étais contente de lire, dans le compte rendu de la rencontre du 28 octobre 2015, que cette notion de malaise avait été exprimée par d'autres personnes; cela me reliait à l'expérience que j’avais vécue au sein de mon implication bénévole en plus de me lier aux autres membres de la communauté de pratique. Pour moi, il était clair qu'il fallait ressentir un malaise, qu'il soit issu d'un conflit de valeurs ou d'un conflit d'intérêts, avant d'amorcer une démarche éthique. C'est comme un déclic physique. C'est pourquoi on voit la procédure Ressentir un malaise trôner fièrement dans le coin gauche supérieur de la figure 2 .

De la même manière, je supposais que mes valeurs personnelles et professionnelles de même que le besoin de maintenir l'équilibre organisationnel en matière de pouvoir, d'autorité et d'objectivité allait régir, voire conditionner le démarrage d'une démarche éthique. En m'inspirant des comptes rendus, j’ai pu identifier d'autres éléments importants qui viennent aussi influencer l'amorce d'une démarche éthique :
- Les défis de gouvernance pour l'atteinte de la mission de l'organisation, en fonction d'une certaine vision et d'une culture organisationnelle;

- Les défis administratifs et les méandres dans lesquels nous nous perdons pour maintenir l'équilibre afin de permettre à tous d'accomplir leurs tâches;

- Les défis politiques, qui sont à granularité variable selon le lieu de leur apparition: équipe, service, faculté ou domaine, organisation, regroupement régional ou provincial;

- Les perceptions qui varient selon les domaines et les contextes, les mœurs et les habitudes;

- Les finalités, politiques ou sociétales, qui orientent la prise de décision et la profusion des services publics/organisationnels.

À tout cela s'ajoute un référentiel de connaissances en éthique pour articuler une pensée éthique pour la profession. Il y a plus que le physique de l'emploi. En effet, il ne servirait à rien d'avoir une conscience éthique de tueur en série pour gérer une salle d'opération! Pour moi, à ce moment, il était clair qu'on venait de définir la place de l'éthique au sein d'une organisation en plaçant ces principes comme des étincelles faisant jaillir le feu.

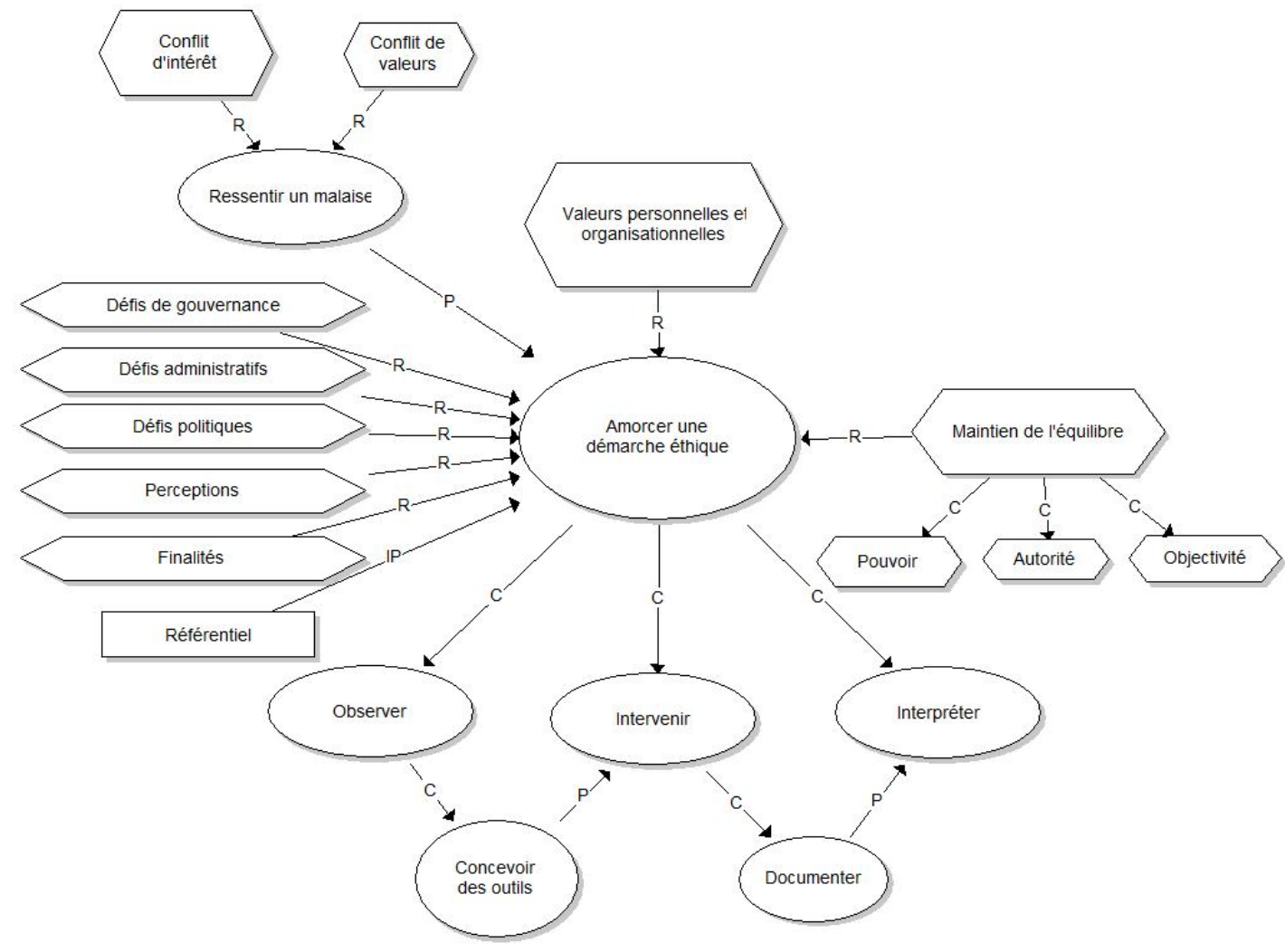

Figure 2 - Premier modèle personnel - Amorcer une démarche 
Une fois qu'on a compris tous ces beaux principes, comment articuler la démarche éthique? Je suis retournée aux contenus des cours du programme court de $2^{\mathrm{e}}$ cycle et j'ai copié et légèrement modifié les étapes du modèle de Ricœur qui s'enroulent sur eux-mêmes sans fin jusqu'à ce que l'on convienne que la résolution du problème éthique est suffisante. Les trois étapes selon Ricœur (1990) sont:

- Observer;

- Intervenir;

- Interpréter.

J'ai intercalé deux étapes qui m'apparaissaient essentielles dont j'avais déjà discuté avec Marc :

- Concevoir les outils;

- Documenter.

Pour Marc, ces deux étapes sont implicites et imbriquées dans les trois premières, mais pour moi, ne connaissant rien de l'éthique, ce n'était pas évident.

\section{Un plongeon vers un modèle en coconstruction}

C'est lors de la 4e rencontre que j'ai présenté le premier modèle d'une démarche éthique au groupe (figure 1). J'en avais discuté auparavant avec Marc et lui avait suggéré que le modèle soit retourné au groupe pour valider l'interprétation que j'avais faite de leurs propos et de ma compréhension. Ça nous ferait un produit final. Il a bondi sur l'idée. J'ai partagé mon écran avec le groupe dans Adobe Connect et j'ai poursuivi la modélisation en direct ou « live » comme diraient nos collègues français! On a discuté tous ensemble de la représentation des connaissances qui ont émergé dans les rencontres précédentes, et en libres penseurs que nous étions, les idées ont foisonné donnant lieu à un nouveau modèle «live », brut plus étoffé (figure 2).

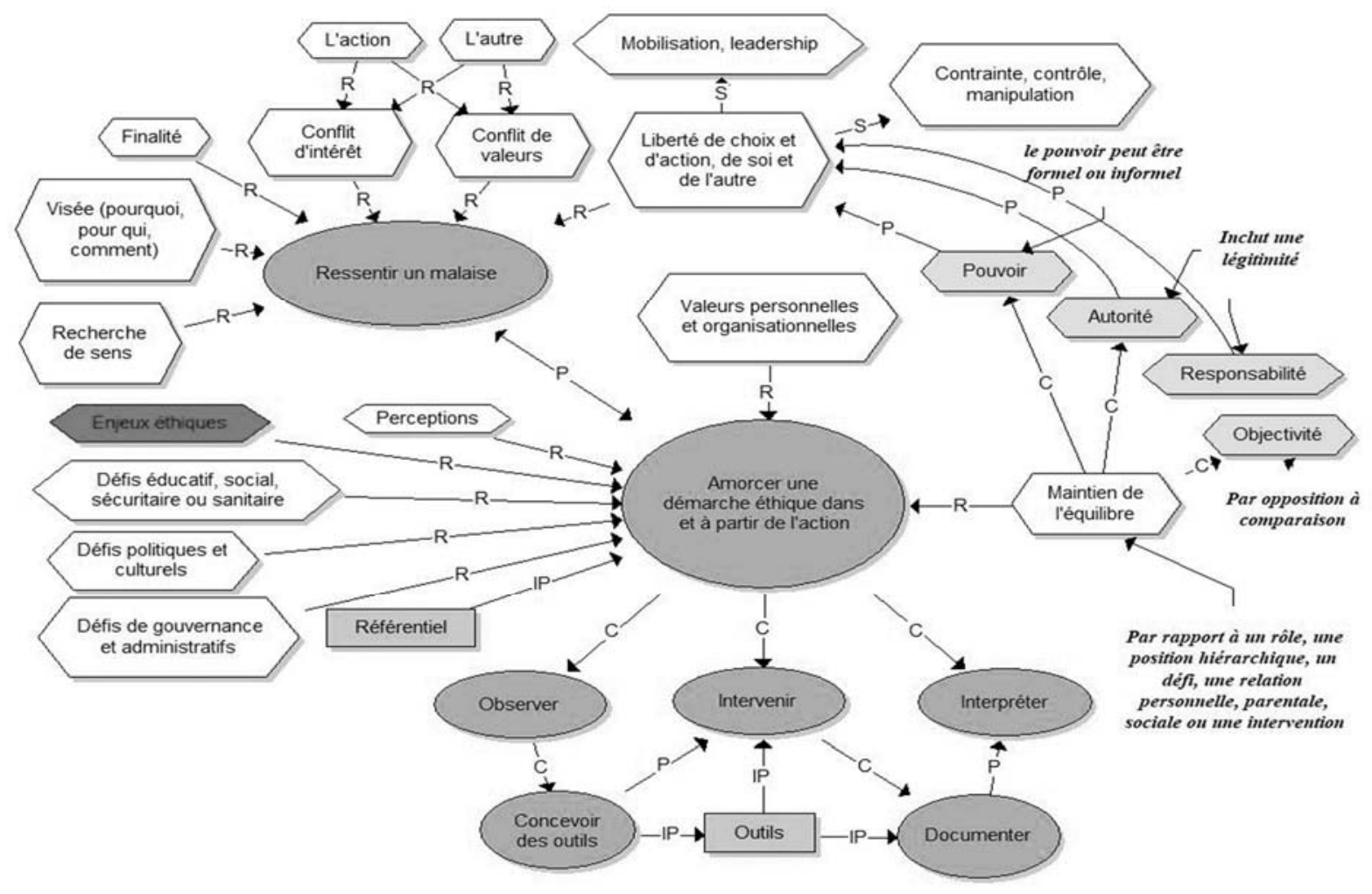

Figure 3 - Deuxième modèle « live » brut (sans correction) 
Dans ce second modèle, on a surtout éclaté les principes qui déclenchent la démarche éthique que nous avons située dans l'action et à partir de l'action. Ressentir un malaise demeure le déclic qui démarre la démarche, que ce soit en fonction de la finalité ou de la visée, de la recherche de sens, de conflits, par rapport à l'action ou à l'autre ou encore par rapport à la liberté de choix ou d'action pour soi ou pour l'autre, dans la mobilisation ou avec leadership, pouvant même mener à la contrainte, au contrôle ou à la manipulation. Je ne comprenais pas que l'on distingue la finalité de la visée jusqu'à ce qu'un membre du groupe suggère qu'elles puissent diverger selon les points de vue personnel et professionnel.

Nous avons aussi précisé que le Maintien de l'équilibre pouvait être par rapport à un rôle, à une position hiérarchique, un défi, une relation personnelle, parentale, sociale ou une intervention qui comprend le pouvoir, l'autorité la responsabilité et l'objectivité. La notion de maintien de l'équilibre inclut celle de légitimité qui s'exprime dans l'autorité et la responsabilité, alors qu'on s'approprie le pouvoir de manière formelle ou informelle. L'objectivité est aussi une composante essentielle du maintien de l'équilibre en considérant chaque élément dans son unicité et s'oppose ainsi à la notion de comparaison.
À la suite de Perceptions, les défis se sont précisés et sont devenus :

- Les défis éducatifs, sociaux, sécuritaires ou sanitaires;

- Les défis politiques et culturels;

- Les défis de gouvernance et les défis administratifs;

- Les conflits.

Je croyais alors que les Conflits, le dernier principe à déclencher une démarche éthique, en bas à gauche, était en conflit avec les Conflit d'intérêt et Conflit de valeurs qui régissent Ressentir un Malaise. Ce n'était pas le cas. Il faut voir ici que Conflit comme un fait qui impose ses principes, c'est-à-dire une obligation. On peut penser à un conflit politique, une guerre, un événement incontournable comme le 11 septembre, etc. Dans le glossaire de MOT, on dirait que Conflits a un autre (A) type de relation avec Amorcer une démarche éthique. Il apparaîtra donc ainsi dans le modèle final :

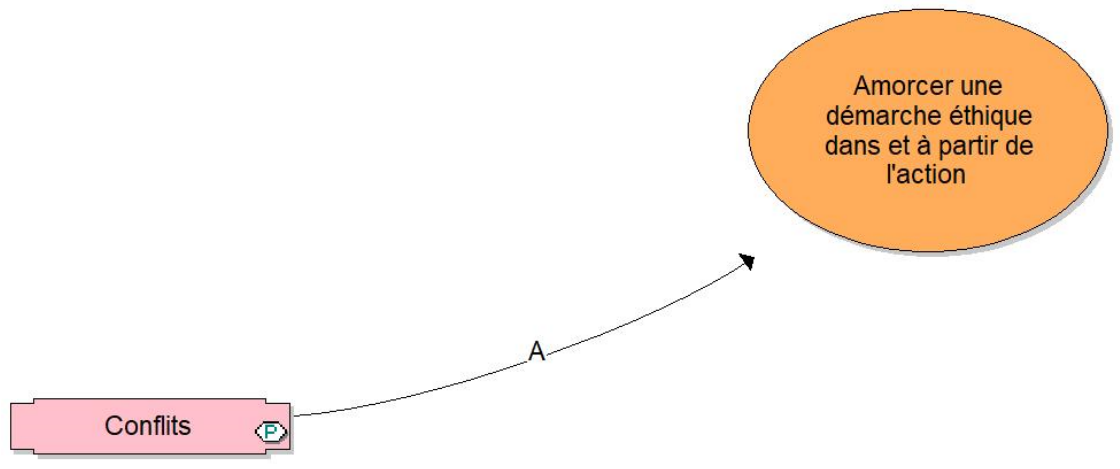

Figure 4 - L'incontournable « Conflits »

Le Référentiel de connaissances en éthique et les Outils sont les deux concepts du modèle et ils sont nécessaires pour amorcer une démarche éthique. Le référentiel de connaissance n'est pas formel et il fait partie des acquis pour la personne qui amorce une démarche éthique. Quant aux outils, ils se définissent lors de la démarche et sont utiles pour intervenir et documenter la démarche. Finalement, le dernier élément du modèle coconstruit est Enjeux éthiques, car des enjeux de 
ce type peuvent évidemment concourir au démarrage d'une démarche éthique.

\section{L'ajout de Ricœur et la dynamisation de la démarche}

Entre la $4^{\mathrm{e}}$ et la $5^{\mathrm{e}}$ rencontre, j'ai peu travaillé sur le modèle, sinon que d'y mettre de la couleur pour harmoniser et mieux distinguer les connaissances. Je l'ai rapporté ainsi à la $5^{\mathrm{e}}$ rencontre. C'est lors de cette dernière rencontre en conférence Web qu'un membre du groupe a constaté que nous avions en quelque sorte regroupé le "Je" de Ricœur autour de Ressentir un malaise. Un autre a renchéri en disant que dans le coin supérieur droit, il retrouvait le « $\mathbf{I} 1$ » de Ricœur. Marc a poursuivi en situant le « $\mathbf{T u}$ » de Ricœur auprès des défis et le Nous auprès du Maintien de l'équilibre. Et puis, tous ensemble, nous avons ajouté le "Je " du groupe, le « $\mathbf{T u}$ » du groupe et le « $\mathbf{I} \mathbf{l}$ » $\mathbf{d u}$ groupe pour intégrer la notion de collectivité et de collaboration au modèle de la démarche éthique. Pour ma part, je n'ai pu que me rallier, car je n'ai toujours pas lu l'œuvre de Ricœur!

Le modèle final de l'équipe québécoise prenait forme (figure 5). Mais il manquait encore quelque chose. Le modèle manquait de vie! En effet, une démarche éthique est dynamique et elle se vit dans l'action; de plus, elle génère de l'énergie à son tour, comme nous l'avions décrit dans le curriculum du programme court de $2^{\mathrm{e}}$ cycle. Lorsque je veux rendre quelque chose dynamique dans un modèle de connaissances à des fins pédagogiques, je mets un lien de composition $(\mathbf{C})$ en boucle sur la connaissance, de sorte qu'on puisse dire qu'on réinvestit dans les processus, les procédures ou les actions qui s'enroulent sur eux-mêmes. On appelle ce lien «boucle de rétroaction». C'est lors d'une rencontre de travail avec Marc et Didier que j'ai soulevé ce point et nous avons convenu d'ajouter des boucles de rétroaction (C) à Observer, Intervenir et Interpréter.

Et puis, soudainement, il y a eu comme un grand vide : le modèle était fini; nous n'avions rien à $\mathrm{y}$ ajouter. C'est alors que Didier et Marc m'ont demandé de participer à l'ouvrage qu'ils souhaitaient écrire... Une chose en entraînant une autre...

\section{Conclusion}

Dans cet article, je vous ai raconté l'histoire de la coconstruction d'un modèle d'une démarche éthique, selon mon point de vue de designer pédagogique, mais aussi de citoyenne s'impliquant dans sa communauté et gestionnaire de service qui tente de comprendre ce qui amorce et régit une telle démarche et tente d'en préciser les composantes et les balises pour mieux encadrer ses décisions au quotidien. Le modèle a émergé de ce désir de compréhension pour devenir une production commune plus grande que la somme de nos connaissances individuelles sur l'éthique.

En tant que gestionnaire dans le domaine public, il m'arrive souvent de vivre des situations qui relèvent de l'éthique. En fait, quand on gère une équipe, j'ai l'impression qu'on gère des éthiques qui diffèrent selon les individus, les sujets et les moments. On entreprend une démarche éthique pour le bien de l'organisation ou pour le bien des personnes impliquées, malgré qu'il s'agisse souvent d'une démarche inconfortable dans laquelle on se marche sur le cœur. Par contre, je constate que le modèle que nous avons collectivement déployé s'applique pour tous, à tout et tout le temps. Ça fait plusieurs fois que je le valide dans des situations des plus diversifiées et chaque fois, je constate que c'est une démarche naturelle et bienveillante, ce qui jette un peu de baume sur mes plaies de gestionnaire et me donne un peu plus de courage managérial pour passer à travers les épreuves de l'appareil public.

Je ne dis pas que c'est un modèle parfait, mais je prétends que c'est un modèle valide qui peut servir de balises et aider à situer un problème éthique en permettant de mettre le doigt sur le bon bobo. Où est-ce que ça fait mal? C'est un problème de pouvoir? Une recherche de sens? On a usurpé votre autorité? Une fois que les sources du malaise sont identifiées, la démarche coule de source elle aussi.

Finalement, je tiens à remercier les professeurs Marc Jean et Didier Benoit et les membres de la communauté de pratique éthique québécoise: Stéphane Claveau, Caroline Fortin, Julie Fortin, Denis Garneau, Sara-Jeanne Lemieux, Johny Perron et Maxim Tremblay. Sans vous, je ne me serais jamais posée autant de questions! Bonne démarche éthique! 


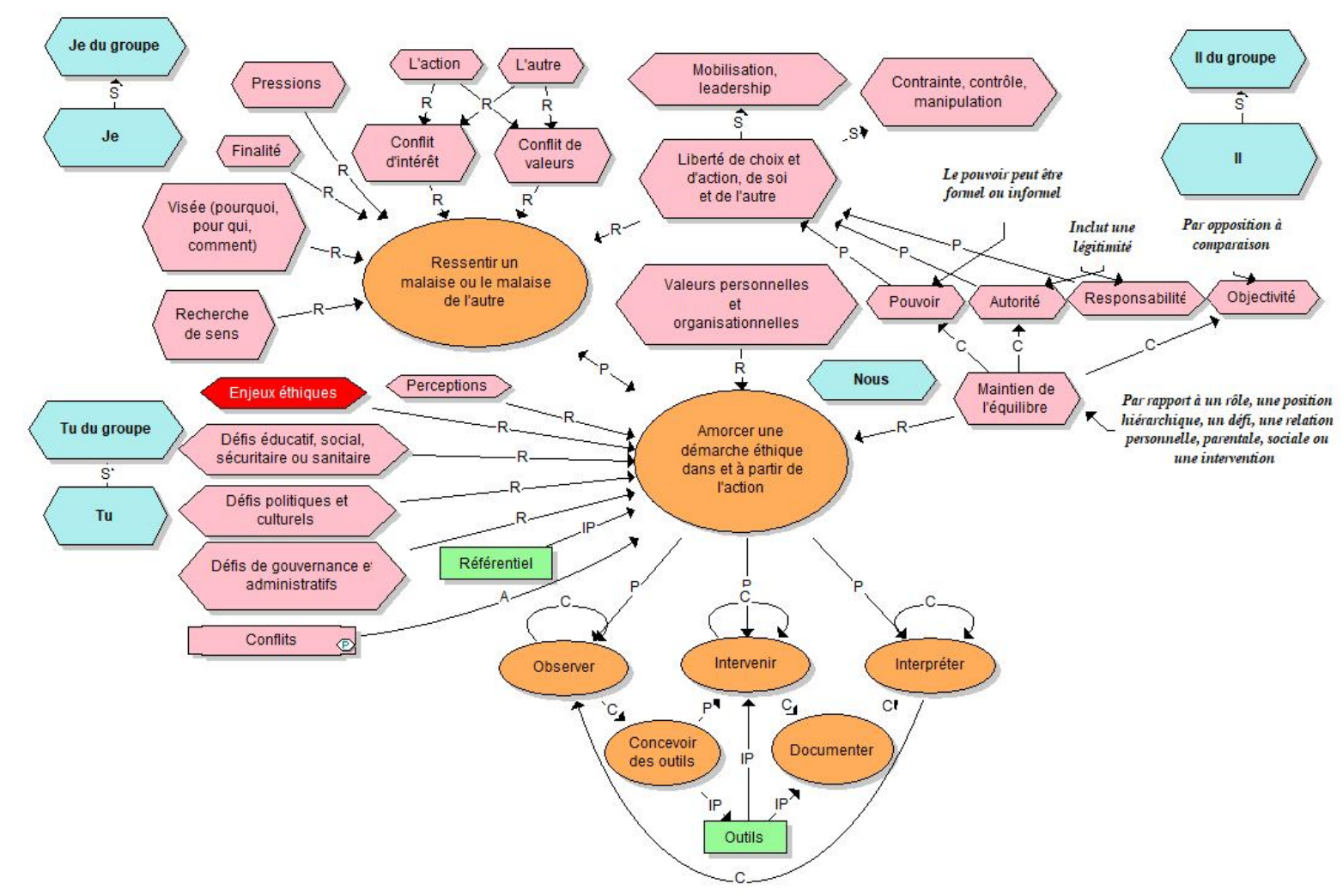

Figure 1- Modèle québécois d'une démarche éthique

\section{NOTES}

1 http://lice.licef.ca/index.php/mot-et-motplus-telechargements/

\section{RÉFÉRENCES}

Ricœur, P. (1990). Soi-même comme un autre. Paris, France : Éditions du Seuil. 